\title{
An Audit of 204 Histopathology Reports Over Three Years of Carcinoma of Cervix: Experience from a Tertiary Referral Centre
}

\author{
Anuja Prakash Pradhan, Santosh Menon, Bharat Rekhi, Kedar Deodhar*
}

\begin{abstract}
Background: The aim was to see compliance to minimum data set information in carcinoma cervix histopathology reports from a team of 13 pathologists; and also to analyse the distribution of parameters like tumor size, grade, depth of cervical stromal invasion, lymph node yield and pTNM stage. Materials and Methods: All pathology reports of radical hysterectomy for carcinoma cervix operated in house within a three year duration (2010-2012), $(n=204)$ were retrieved from medical records and analyzed for the above parameters. Results: In 2010- 59 cases, in 2011- 67 cases and in 2012- 78 cases of carcinoma cervix underwent operations in our hospital. The median age was 50.5 years and the maximum $T$ diameter was $2.8 \mathrm{cms}$ in the reports of three years. Squamous carcinoma was the commonest subtype amongst all the tumors. It was noted that $60.8 \%$ of cases had cervical stromal involvement more than half the thickness of the cervical stroma. Parametrial involvement was seen in $4.82 \%$ of cases. pTNM Staging was not mentioned in $65.06 \%$ of the cases. The mean bilateral pelvic lymph node yield count in our study was 16.6 inclusive of all the three years. Conclusions: Compliance with provision of a minimum dataset in our team of 13 pathologists was generally good. Lymph node yield in our hands is reasonable, but constant striving for greater numbers should be made. pTNM staging should be more meticulously documented. Use of proformas /checklists is recommended.
\end{abstract}

Keywords: Cervical carcinoma - audit - histopathology report - three years

Asian Pac J Cancer Prev, 16 (14), 5643-5645

\section{Introduction}

Histopathological reporting of surgically resected cancer specimens requires specific information in the reports for confirmation, extent and stage of the tumor. This also helps determine the adjuvant therapy and also helps in the quality control exercise of other departments (e.g. Surgery, Radiology).The proformas (minimum datasets) are widely and freely available on websites of reputed international organizations (e.g. Royal College of Pathologists-RCPath, College of American PathologistsCAP).

The aims and objectives of the study were as follows:

1) To verify whether all the relevant parameters deemed important in deciding further management of a patient are mentioned in the Histopathology reports (compliance to minimum data sets).

2) To analyse the distribution of various parameters like tumor type, depth of cervical stromal involvement, mean lymph node yield etc. which measure the completeness of pathology examination.

\section{Materials and Methods}

All pathology reports of in-house operated cases of carcinoma cervix/Radical hysterectomy of three years duration (January 2010 - December 2012) were retrieved from Pathology records. A total of 204 inhouse operated cases were included for this study. A team of 13 pathologists report these cases (in rotation), and a proforma for carcinoma cervix is used. We use a free text proforma which is broadly based on RCPath and CAP Datasets. Following parameters were recorded from the reports: age, tumor size, tumor type, tumor differentiation, involvement of parametrium, vagina, vaginal cut margins, depth of cervical stromal invasion and involvement of cervical adventitial cut margin, Presence of lymphovascular emboli and perineurial invasion, Bilateral lymph nodal yield.

The specimens from the operation theatre are sent to our department in fresh state in plastic sealed bags. The specimens are opened and fixed overnight in $10 \%$ buffered formalin and dissected in fixed state as per the standard protocol. Lymph nodes are also dissected along with the 
main specimen. WHO Classification for subtyping the cervical carcinomas is used.

\section{Results}

A total of 204 cases of carcinoma cervix were operated in our hospital from January 2010-December 2012. The median age was 50.5 and the median maximum $\mathrm{T}$ size was $2.8 \mathrm{~cm}$.

The subtype of the tumor, differentiation was mentioned in all the reports. Squamous carcinoma was the commonest subtype amongst all the tumors. Table 1 gives in detail the different histopathological parameters studied and their distribution.

Lymphovascular emboli (LVI), perineurial invasion, vaginal and parametrial involvement are important components of the report and these were mentioned in all the reports $(100 \%)$.

LVI is one of the best prognostic factors for diseasefree and overall survival (Sykes, 2003).Even in the absence of nodal involvement, LVI is deemed a strong prognostic factor and it correlates with other prognostic markers such as tumor type and stage (Sykes, 2003).

Depth of cervical stromal invasion was mentioned in all the reports. It was noted that $60.84 \%$ of cases had cervical stromal involvement more than half the thickness of cervical stroma.

The mean, median and range of bilateral pelvic nodes dissected in the three years was studied. For lymph node retrieval, careful palpation and separation is followed; fat retrieval solutions are not used, or processing of entire fat is not processed.

The overall lymph node yield was found to be

Table 1. Parameters that were Studied

\begin{tabular}{lrr}
\hline Type of Carcinoma(n=204) & No. & $\%$ \\
\hline Squamous carcinoma & 146 & $71.60 \%$ \\
Adenocarcinoma(NOS) & 10 & $4.90 \%$ \\
Adenosquamous carcinoma & 4 & $1.96 \%$ \\
Basaloid Squamous carcinoma & 1 & $0.49 \%$ \\
Endometrioid adenocarcinoma & 2 & $0.98 \%$ \\
Lymphoepithelioma like & 1 & $0.49 \%$ \\
Mesonephroid adenocarcinoma & 1 & $0.49 \%$ \\
Clear cell adenocarcinoma & 1 & $0.49 \%$ \\
CIN 3 & 20 & $9.80 \%$ \\
No residual tumor (Post chemotherapy) & 17 & $8.33 \%$ \\
Chronic cervicitis & 1 & $0.49 \%$ \\
Parameters(n=166) & No & $\%$ \\
Involvement of vagina & 9 & $5.42 \%$ \\
Involvement of vaginal cut margin & 2 & $1.20 \%$ \\
Involvement of parametria & 8 & $4.82 \%$ \\
LVI present & 50 & $30.10 \%$ \\
PNI present & 9 & $5.42 \%$ \\
Nodal metastasis & 24 & $14.40 \%$ \\
Cervical stromal involvement(n=166) & No & $\%$ \\
Less than 1/2 cervical stromal involvement & 54 & $32.50 \%$ \\
1/2 cervical stromal involvement & 11 & $6.63 \%$ \\
More than 1/2 cervical stromal involvement & 101 & $60.80 \%$ \\
B/L Nodal parameters(n=204) Combined years $2010-2012$ \\
Mean B/L Pelvic lymph node yield & 16.6 \\
Median B/L Pelvic lymph node yield & 16 & \\
Range of B/L Pelvic lymph node yield & $4-39$ & \\
\hline & \multicolumn{3}{c}{} \\
\hline
\end{tabular}

increasing in the subsequent years denoting a better sample quality and diligent grossing practices. (Mean bilateral pelvic lymph nodal yield-14.05 in 2010, 17.69 in 2011 and 18.04 in 2012)

\section{Discussion}

Audit is a systematic and independent examination to determine whether quality activities and related results comply with the planned arrangements, and whether these arrangements are implemented effectively and are suitable to achieve the objectives (1991).

In this audit exercise, we have assessed the completeness of the final report with regard to minimum datasets. These minimum datasets (macroscopic and microscopic findings) for all the cancers are available. The utility of audits in pathology is well known. Audits, even in developed countries, can bring out the inadequacies in the histopathology reports. However, it is well recognized that standardization in cancer reporting improves the completeness and quality of the reports. With respect to similar audits in our part of the world, we came across, an audit of rectal carcinoma reported by Nambiar et al (Nambiar et al., 2010)from India and breast carcinoma by Mamoonet al (Mamoon et al., 2010)from Pakistan.

In general we use a proforma report/checklist necessary for cancer reporting which is based on RCPath (Royal College of Pathologists,UK) .However our reports are written in free text form and not in the form of ticking or filling in the blanks.

The above findings show that the compliance to adhere to minimum data information in a team of 13 pathologists is generally good. This is not a statistical statement but a measured overall impression based on our findings.

Tumor type,tumor differentiation, involvement of parametrium,vagina, vaginal cut margins, depth of cervical stromal invasion and involvement of cervical adventitial cut margin, presence of lymphovascular emboli and perineurial invasion, bilateral lymph nodal yield was mentioned in all the reports.

Cervical stromal infiltration-An additional parameter that has been reported to be of prognostic significance in cervical carcinomas is the depth of infiltration in thirds of the cervical wall (Delgado, 1990)Tumor depth correlated strongly with disease-free interval whether measured in absolute terms $(\mathrm{mm})$ or in fractional thirds as for corpus cancers (Delgado, 1990). Depth was found to be an independent prognostic factor in node-negative patients with stage Ib and II cervical cancer (Samlal and Hart, 1997)

Parametrial involvement-This is a poor prognostic factor in early stage cervical cancer, regardless of lymph node status (Zreik et al., 1996). It also correlates significantly with pelvic nodal metastasis, uterine and vaginal involvement in early stage IA-IB cancers. (Chatchotikawong, 2014)

In surgically treated stage II carcinomas, the extent of parametrial involvement is an important prognostic indicator (Kamura, 1999; Aoki, 2000; Takeda, 2002). Parametrial involvement was seen in $4.82 \%$ of all the cases in the three years. 
An Audit of 204 Histopathology Reports over Three Years of Carcinoma of Cervix: Experience from a Tertiary Referral Centre

Vaginal Surgical Margin-In early stage cervical cancers microscopic positive vaginal surgical margin is a high risk pathological factor for decreased survival and increased recurrence. The correlation of vaginal mucosa involvement by High grade squamous intraepithelial neoplasia (HSIL) and a positive vaginal cut margin was studied and it was found to be significant. (Sethasathien S, 2014) Vaginal cut margin involvement by malignancy was $1.2 \%$ in our study.

pTNM Staging-This was not mentioned in close to $65.06 \%$ of the cases in all the three years. Histopathology is important in staging stage I cancers, in the measurement of tumors that are not visible macroscopically.

Lymphovascular emboli- The presence or absence of lymphovascular emboli must be documented. However, their presence does not upstage the tumor. Their presence may warrant adjuvant chemotherapy.

Lymph nodes- The minimum number of lymph nodes to be examined, (e.g. 12, in surgical specimens for colorectal carcinoma) is not defined in the literature for cervical carcinoma. Pieterse et al (Pieterse et al., 2007) reported that the disease-free survival (DFS) of nodepositive patients with $\geq 18$ removed lymph nodes was $20 \%$ higher than that of patients with $<18$ removed lymph nodes. Prapaporn et al. suggested that more than 10 nodes should be removed for standard pelvic lymphadenectomy (Prapaporn S, 2012). Similarly, removal of at least 12 pelvic lymph nodes was suggested as a quality indicator for pelvic lymphadenectomy in an European Organisation for Research and treatment of cancer-Gynaecological cancer group (EORTC-GCG) study. (Verleye, 2009) According to the International Union against Cancer, a minimum of 10 lymph nodes should be investigated for the determination of pN0 (Horn, 2005). The mean bilateral pelvic lymph node yield count in our study is $14.05,17.69$ and 18.04 in 2010, 2011 and 2012 respectively.

For early stage cancers, high risk pathological factors that connote a worse prognosis in terms of recurrence and survival have been stated. These include a positive surgical margin, parametrial involvement and pelvic nodal metastasis. These become candidates for adjuvant pelvic irradiation with or without chemotherapy. (Randall)

In conclusion, i) A meticulous report is important as it determines the treatment and prognosis. It also facilitates regular audits and review of all aspects of services; $i$ ) Compliance to adhere to minimum dataset in our team of 13 pathologists is generally good; iii) Squamous carcinoma is the commonest type of cervical carcinoma; iv) Lymph node yield in our hand is good, but a constant vigil for more number should be made; v) pTNM Staging should be more meticulously mentioned; vi) Use of proformas/checklists is very helpful in not missing out on a parameter and hence is recommended.

\section{References}

Aoki Y SM, Watanabe M, et al (2000). High-risk group in node-positive patients with stage IB, IIA, and IIB cervical carcinoma after radical hysterectomy and postoperative pelvic irradiation. Gynecol Oncol, 77, 305-9.

Chatchotikawong U,Ruengkhachorn I, Leelaphatanadit C
(2014). Residual disease following conization of women with stage IA-IB1 cervical carcinoma in a high incidence region. Asian Pac J Cancer Prev, 15, 7383-87.

Delgado G BB, Zaino R, Sevin BU, Creasman WT, Major F (1990). Prospective surgical pathological study of diseasefree interval in patients with stage IB squamous cell carcinoma of the cervix: a Gynecologic Oncology Group study. Gynecol Oncol, 38, 352-7.

Horn LC EJ, Hockel M, et al (2005). [Recommendations for the handling and oncologic pathology report of lymph node specimens submitted for evaluation of metastatic disease in gynecologic malignancies]. Pathologe, 26, 266-72.

Kamura T ST, Kaku T et al (1999). Histopathological factors influencing pelvic lymph node metastases in two or more sites in patients with cervical carcinoma undergoing radical hysterectomy. Acta Obstet Gynecol Scand, 78, 452-7.

Mamoon N, Hassan U, Mushtaq S, et al (2010). Histopathology of breast carcinoma-an audit of 50 Reports in Rawalpindi, Pakistan. Asian Pac J Cancer Prev, 11, 169-72.

Nambiar A, Vivek N, Bindu M, et al (2010). Completeness of low anterior resection pathology report: A hospital-based audit with recommendations on improving reporting. Indian $J$ Cancer, 47, 156-9.

Pieterse Q, Kenter G, Gaarenstroom K, et al (2007). The number of pelvic lymph nodes in the quality control and prognosis of radical hysterectomy for the treatment of cervical cancer. Eur J Surg Oncol, 33, 216-21.

Prapaporn S KC, Surapan K (2012). Pelvic node removal and disease-free survival in cervical cancer patients treated with radical hysterectomy and pelvic lymphadenectomy. Int $J$ Gynecol Obstet, 116, 43-6.

Randall ME FP, Toita T et al (2013) Principles and Practice of gynecologic oncology, Philadelphia, Lipincott Williams and Wilins.

Samlal RA vdVJ, Ten Kate FJ, Schilthuis MS, Hart AA LF (1997). Surgical pathologic factors that predict recurrence in stage IB and IIA cervical carcinoma patients with negative pelvic lymph nodes. Cancer, 80, 1234-40.

Sethasathien S, Charoenkwan K, Settakorn J, Srisomboon J. (2014). Predicting factors for positive vaginal surgical margin following radical hysterectomy for stage IB 1 carcinoma of the cervix. Asian Pac J Cancer Prev, 15, 2211-15.

Sykes PAD, Cohen C, Scurry J, Yeo D (2003). Does the density of lymphatic vascular space invasion affect the prognosis of stage Ib and IIA node negative carcinoma of the cervix? Int J Gynecol Cancer, 13, 313-6.

Takeda N SN, Takeda M et al (2002). Multivariate analysis of histopathologic prognostic factors for invasive cervical cancer treated with radical hysterectomy and systematic retroperitoneal lymphadenectomy. Acta Obstet Gynecol Scand, 81, 1144-51.

Verleye L VI, Reed N, Ottevanger PB (2009). Quality assurance for radical hysterectomy for cervical cancer: the view of the European organization for research and treatment of cancer-gynecological cancer group (EORTC-GCG). Ann Oncol, 20, 1631-8.

Zreik TG, Chambers JT, Chambers SK (1996). Parametrial involvement, regardless of nodal status: a poor prognostic factor for cervical cancer. Obstet Gynecol, 87, 741-6. 